\title{
A Psychometric Analysis of the Workplace Forgiveness Scale
}

\author{
Itsara Boonyarit ${ }^{\star a}$, Wiladlak Chuawanlee ${ }^{b}$, Ann Macaskill $^{c}$, Numchai Supparerkchaisakul ${ }^{b}$
}

[a] Division of Psychology, Chiang Mai University, Chiang Mai, Thailand. [b] Behavioral Science Research Insititute, Srinakharinwirot University, Bangkok, Thailand. [c] Psychology Research Group, Sheffield Hallam University, Sheffield, United Kingdom.

\begin{abstract}
Though there are measures of forgiveness published in the behavioural science literature, very few scales are available to measure forgiveness in workplace relationships. The Workplace Forgiveness Scale aimed to measure forgiveness of a specific offense. Data from 348 professional nurses in Thailand were subjected to an exploratory factor analysis (EFA) and the psychometric properties of the scale were examined. Results from EFA suggested retaining four underlying factors of the forgiveness construct: Overcoming Negative Thought and Feeling toward the Offender, Seeking to Understand the Offender's Reasons, Fostering Positive Approaches towards the Offender, and Belief in the Benefits of Forgiveness. Reliability coefficients for the total scale and subscales were adequate. Evidence of construct validity is presented. Scores on the forgiveness scale were positively associated with other related forgiveness constructs. Nomological validity analysis supported the theoretical networks of the forgiveness construct. Forgiveness played the complete mediating role in the relationship between dispositional forgiveness and willingness to reconcile, and played a partial mediating role in the relationship between rumination and seeking to revenge the offender. Bootstrap analysis on the parameter estimates of the sample results revealed satisfactory level of internal replicability and stability of the results across the samples. Implications for future research are discussed.
\end{abstract}

Keywords: forgiveness, scale development, work context, construct validity

Europe's Journal of Psychology, 2013, Vol. 9(2), 319-338, doi:10.5964/ejop.v9i2.551

Received: 2012-11-15. Accepted: 2013-04-07. Published: 2013-05-31.

${ }^{*}$ Corresponding author at: Division of Psychology, Faculty of Humanities, Chiang Mai University, Chiang Mai, Thailand 50200. E-mail: dr.itsara@gmail.com

This is an open access article distributed under the terms of the Creative Commons Attribution License

(http://creativecommons.org/licenses/by/3.0), which permits unrestricted use, distribution, and reproduction in any medium, provided the original work is properly cited.

Forgiveness is one of the constructive ways of moderating workplace conflict and encouraging more positive cooperative behaviours (Butler \& Mullis, 2001). An individual who practises forgiveness as a problem-solving strategy can reduce anger, revenge, and negative judgment towards the offender (McCullough \& Worthington, 1994). Forgiveness should be addressed by organizational theorists and practicing managers as it is important for individuals to be facilitated in order to repair broken relationships with work colleagues (Aquino, Grover, Goldman, \& Folger, 2003). Forgiveness is also associated with better health and personal well-being at the individual level (McCullough \& Witvliet, 2002) and, at the organizational level, forgiveness essentially encourages a more nurturing and fulfilling workplace climate (Stone, 2002).

Although the theoretical concept and empirical basis of forgiveness have been investigated substantively since the 1980s (McCullough, Pargament, \& Thoresen, 2000), within the management and organizational literature, there is very little research on forgiveness within the workplace (Aquino et al., 2003; Cameron \& Caza, 2002). To understand forgiveness in the work context is a complex undertaking, and questions remain to be investigated (Madsen, Gygi, Hammond, \& Plowman, 2008). McCullough et al. (2000) mentioned that many aspects of forgiveness cannot be examined empirically because the measuring scales have not yet been constructed in many sociocultural contexts, such as work and organizational settings which include specific cultural factors. This study aimed to address this by developing a workplace forgiveness scale within Thai culture, beneficial for allowing further study of forgiveness in workplace relationships. 


\section{The Current Study}

To achieve a good quality scale to measure forgiveness within the work context, as advised by Kline (1986), we used knowledge derived from the research literature on forgiveness in a work context and empirical evidence in the form of definitions of the forgiveness process collected from a previous qualitative study of forgiveness among Thai nurses in their workplace (Boonyarit, Chuawanlee, Macaskill, \& Supparerkchaisakul, 2012). These sources were used to produce the initial items for the Workplace Forgiveness Scale. The pool of items was then subjected to exploratory factor analysis to determine the underlying factor structure (Costello \& Osborne, 2005; Fabrigar, Wegener, MacCallum, \& Strahan, 1999) and the internal replicability was investigated to establish the invariance of the factors across samples (Timmerman, Kiers, \& Smilde, 2007; Zientek \& Thompson, 2007). Finally, construct validation was employed to determine the convergent, discriminant, and nomological validity of the forgiveness construct using other related constructs (Cronbach \& Meehl, 1955; Hair, Black, Babin, Anderson, \& Tatham, 2006).

\section{Construction of the Initial Workplace Forgiveness Scale}

The Concept of Forgiveness Within the Workplace. Forgiveness is a willingness to discard one's right to revenge and instead to show mercy to the offender (Enright \& Coyle, 1998). It is a motivation to reduce avoidance of the offender, as well as to abandon any anger, grudge holding, or revenge towards the offender; conversely, it helps to increase conciliation when the moral norms can be re-established (Worthington, 1998). McCullough et al. (2000) concluded that forgiveness is an intraindividual, prosocial change toward the offender that occurs within a specific interpersonal relationship. In the organizational context there are several definitions of forgiveness. Aquino et al. (2003) suggested that interpersonal workplace forgiveness is a process where the individual, who was hurt by his or her colleague, attempts to overcome negative feelings - such as resentment and anger - toward the offender and to stop himself or herself from causing the offender harm even if he or she believes it is ethically justifiable to do so. While Aquino et al. (2003) focused on the individual level of the forgiveness process, Cameron and Caza (2002) defined forgiveness more broadly at an organizational level. They presumed that organizational forgiveness is the capacity to encourage collective abandonment of justified resentment, hurt, and blame. Moreover, it is the fostering of constructive, forward-looking ways in response to broken relationships. This process requires a transformation resulting in the organization becoming more virtuous.

Previous qualitative research in a work context with Thai nurses (Boonyarit et al., 2012) identified five dimensions defining forgiveness. These were:

- Overcoming negative approaches towards the offender, where the individual attempts to cut off or control their potential oppositional acts towards the offender by overcoming negative thinking and emotions;

- Abandonment of negative judgment, where the individual seeks to understand the offender's reasons, does not categorize the offense as a wrongful act, accepts the offender's mistake, takes the offender's perspective, and abandons negative judgment;

- Fostering positive approaches and loving-kindness towards the offender, where the individual promotes or motivates positive responses to the offender by fostering positive thinking, fostering positive emotions such as empathy and good feeling, and continues to act in a friendly manner towards the offender;

- Awareness of the benefits of forgiveness, the individual is aware that forgiveness leads him/her to happiness and forgiveness would potentially lead to forgiveness in return from the offender;

- Forgiveness as Buddhist beliefs, where the individual believes in the Buddhist teachings that forgiveness is the higher-order merit of giving and is good Karma. 
Characteristics of the Workplace Forgiveness Scale. While there are several psychometrically sound scales measuring forgiveness such as Wade's Forgiveness Scale (Wade, 1987), Transgression-Related Interpersonal Motivation (TRIM: McCullough, Rachel, Sandage, Worthington, Brown, \& Hight, 1998), and the Enright Forgiveness Inventory (EFI: Subkoviak, Enright, Wu, Gassin, Freedman, Olson, \& Sarinopoulos, 1995), these have all been developed with North American samples and their applicability to different cultures is debatable. The aim here is to develop a psychometrically sound scale that would measure forgiveness within a Buddhist culture, specifically incorporating Buddhist concepts such as loving kindness, higher-order merits and Karma. All of these concepts were shown to be relevant to forgiveness in previous research on work place conflict in a sample of Thai nurses (Boonyarit et al., 2012).

Items and Rating Scale. The pool of initial items was designed to measure forgiveness towards a specific offender within a work-related offense. A forty-item scale was developed based on the five dimensions of forgiveness (Boonyarit et al., 2012); it conceptualized forgiveness as the individual's cognitive, affective, and behavioural responses towards the offender. The scale instructed the respondents to choose the answer that best described their thoughts towards the person who has hurt or mistreated them in the past by using a Likert-type format with response. Higher scores on this scale represent greater forgiveness towards an offender.

Content Validity. The three content experts chosen included a scholar in behavioural science research, an expert in industrial and organization psychology, and an expert in nursing science. They were briefed on the purpose of the Workplace Forgiveness Scale and were asked to provide feedback on the initial forgiveness scale items. The criteria for item revision included: a) congruence with the relevant definition of the forgiveness from Boonyarit et al. (2012), b) item clarity, c) relevance for the intended population of Thai nurses and their work setting. We then revised the scale in light of the feedback received before data collection began.

\section{Method}

\section{Participants}

The sample was drawn by cluster random sampling from professional nurses who work in three hospitals located in the Bangkok metropolitan and the surrounding area of about 100 kilometres in Thailand. We collected data in various clusters of operational units. The directors of the hospital and the supervisors of the nursing departments gave permission for data collection. Questionnaires were sent to the participants with an introductory covering letter. Returning the questionnaire was accepted as providing informed consent. Sample size was determined by using five times the number of scale items as suggested by Gorsuch (1983). With 40 items in the initial at least 200 participants were required. The participants were 348 full-time nurses in Thailand and consisted of 305 (87.64\%) female, $20(5.75 \%)$ male, and $23(6.61 \%)$ no response. The age included $67(19.25 \%)$ less than 25 years old, 104 (29.89\%) between 25 and 29 years old, 86 (24.71\%) between 30 and 35 years old, 35 (10.06\%) between 36 and 40 years old, $20(5.15 \%)$ between 41 to 45 years old, $12(3.45 \%)$ over 45 years old, and 24 $(6.50 \%)$ no response. The breakdown of participants by unit was $67(19.25 \%)$ surgery, $61(17.53 \%)$ general medicine, 51 (14.66\%) inpatient service, 28 (8.05\%) intensive care unit, 21 (6.03\%) obstetrics and gynecology, 17 (4.89\%) outpatient service, 16 (4.60\%) emergency, 14 (4.02\%) psychiatry, 13 (3.74\%) pediatrics, $11(3.16 \%)$ health promotion, $9(2.59 \%)$ eye, ear, nose and throat, 4 (1.15\%) orthopedic, and $36(10.34 \%)$ no response. 


\section{Measures}

Measure for Exploratory Factor Analysis. The initial 40 items of the Workplace Forgiveness Scale measured forgiveness towards a specific offender within a specific work-related offense that participants were first asked to describe. The scale then instructed respondents to choose the answer that best described their thoughts, feelings, and actions towards the person who had hurt or mistreated them in the past using a Likert-type scale with responses ranging from 1 (strongly disagree) to 6 (strongly agree). Higher scores on this scale represent greater forgiveness towards the offender.

Measures for Convergent Validity Analysis. Here the aim is to ensure that the questionnaire does measure relevant forgiveness constructs and therefore other standard measures of these constructs were included to examine whether our forgiveness measure correlates with these other similar measures. The presence of moderate to high correlations is taken as evidence of construct validity (Cronbach \& Meehl, 1955). Offense-specific forgiveness was measured by the Forgiveness Scale (Rye, Loiacono, Folck, Olszewski, Heim, \& Madia, 2001). The scale consists of 15 items with two subscales, the absence of negative response and the presence of positive response to wrongdoer. Participants were scored on a Likert-type scale with five rating points from strongly disagree to strongly agree. Higher scores on this scale indicate a greater level of forgiveness towards a specific offender. The Alpha coefficient for this scale in the present study was .83 .

Dispositional forgiveness was measured by six items of the Heartland Forgiveness Scale (Yamhure-Thompson \& Snyder, 2003). This scale intends to capture the likelihood of forgiving others. Items were rated on a 7-points Likert scale from almost always false to almost always true. A higher score on this scale indicates being more likely to forgive the other. The Alpha coefficient for this scale in the present study was .67 .

State forgiveness was measured by a single item developed by the researchers. The scale asked the participants to rate "how much do you forgive the specific offender in your work relationship conflict". The item was rated from 1 (I haven't forgiven at all) to 5 (I have completely forgiven). Higher score on this item indicates a state of forgiveness towards the offender.

Measure for Discriminant Validity Analysis. We examined the discriminant validation of the Workplace Forgiveness Scale with respect to measure of individual's current feelings. Ten items of the International Positive and Negative Affect Schedule (PANAS) Short Form (I-PANAS-SF: Thompson, 2007) were included to assess the positive and negative emotional responses by the individual to certain events. Items were rated on a 5-points Likert scale never to always. Higher scores in the negative items indicate higher negative feelings of the individual, and higher score on positive items indicate higher positive feelings.

Measures for Nomological Validity Analysis. Here the aim is to include items which will allow assessment of how developed items and scale relate to other constructs. This was to confirm that our forgiveness scale behaves in a theoretically expected way, as evidence of construct validity (Viswanathan, 2005). Willingness to reconcile was measured by two items from the Willingness to Reconcile Relationship Scale (Tomlinson, Dineen, \& Lewicki, 2004). These items were "What is the likelihood that you would continue a relationship with him/her?" and "To what degree are you willing to let him/her try to reconcile the relationship with you?." The participants responded on a five-point Likert scale from 1 (least) to 5 (most). Higher scores indicate stronger willingness to reconcile with the offender. The Alpha coefficient for this scale in the present study was .86 . 
Rumination was measured with the Rumination about an Interpersonal Offense Scale (RIO: Wade, Vogel, Liao, \& Goldman, 2008). Six items were used to capture state or situation-specific rumination reflecting repetitive cognitive rehearsal about the specific past transgression. Items were assessed on five-point Likert scale ranging from strongly disagree to strongly agree. Higher scores on this scale indicate strong mental attention to negative experience and the outcome of the event. The Alpha coefficient for this scale in the present study was .88.

Seeking revenge was measured with the revenge subscale of the Transgression-Related Interpersonal Motivations Inventory (McCullough et al., 1998). Five items of the revenge subscale were rated using five point Likert scale ranging from strongly disagree to strongly agree. Higher scores on this scale indicate higher levels of revenge seeking. The Alpha coefficient for this scale in the present study was .95.

Two scales from the convergent validity tests were included in the model examining the nomological network of the forgiveness construct and its related variables. These were the forgiveness scale and dispositional forgiveness.

\section{Data Analysis}

We implemented various data analyses to assure the psychometric of the Workplace Forgiveness Scale. The initial items of the scale were submitted to exploratory factor analysis (EFA) using principal component analysis (PCA) with Varimax rotation to investigate the underlying factor structure (Costello \& Osborne, 2005; Fabrigar et al., 1999). The internal replicability was investigated through a Bootstrap method to indicate the invariance of the factors across the samples internally. Bootstrapped eigenvalues provided by the Bootstrap Factor Analysis proposed by Zientek and Thompson (2007) was conducted to determine the internal replicability of the number of factor to retain and Bootstrap Procrustes Confidence Interval (Timmerman et al., 2007) was conducted to reveal the replicability of factor loadings. Assessment of reliability through cronbach's Alpha coefficient (Cronbach, 1951) and composite reliability (Raykov, 1997) were examined. Three measures of construct validity (Cronbach \& Meehl, 1955; Hair et al., 2006) were implemented, convergent validity, discriminant validity, and nomological validity, to examine the theoretical related properties of the forgiveness construct derived from scale development.

\section{Results}

The preliminary analyses revealed the characteristics of the work-related offensive event. Participants reported almost half of the offenders were their colleagues $(49.43 \%)$, other professions $(21.26 \%)$, doctors $(12.64 \%)$, and their supervisors $(8.05 \%)$, respectively. The most common causes of work-related conflict were role conflict $(20.40 \%)$, misunderstanding (17.82\%), injustice of workload $(12.64 \%)$, performance error $(10.92 \%)$, being new to the task $(9.48 \%)$, personal bias $(7.76 \%)$, difference in profession and work status $(5.75 \%)$, and other causes, such as the offender implying professional incompetence, miscommunication, and offender's improper behaviour $(8.91 \%)$.

\section{Exploratory Factor Analysis}

To begin to develop a psychometrically sound measure, an EFA with Varimax-rotation was computed on the initial 40-item Workplace Forgiveness Scale to identify the number of factors to retain and to examine the quality of the items. Bartlett's (1954) test of sphericity produced a significant chi-square at the .0001 level (Chi-square $=7507.98$, $d f=780$ ) and the measure of sampling adequacy (Kaiser, 1974) was equal to .93, indicating that the correlation matrix from the initial 40-items scale is appropriate for conducting EFA. Parallel analysis (Horn, 1965) was conducted to provide an objective criterion for determining the number of factors to retain. To achieve this analysis, 
we implemented MacParallel (Watkins, 2006) to compute the table of random data eigenvalues using the 40 observed variables and 348 participants as being equal to the sample data. The program generated eigenvalues for a random data set that was compared with eigenvalues from the sample's PCA. A parallel analysis plot of 40 items showed the eigenvalues of the sample data were larger than the eigenvalues from the random data before the fifth factor. As a result, we concluded that four factors should be retained for the initial 40 -items of the forgiveness scale.

To attain interpretable factors, we applied Varimax rotation aiming to find the factor loadings which maximize the higher variance on their primary factors and are lower on the other factors (Tabachnick \& Fidell, 1996). With regard to the four factors extracted on the initial 40-item scale, the rotated factor loadings loaded thirteen items on factor I, four items on factor II, fourteen items on factor III, and nine items on factor IV. The results showed several items aligned on the different factors and were incongruent with the concept of the initial item construction. To maintain both statistical and substantial significance for the new forgiveness scale, the problematic items were eliminated using the following criteria: a) factor loading on the primary factor should be equal or more than .30 (Fabrigar et al., 1999), b) there is no high cross-loading (factor loadings loaded on the other factor should not be more than .30) (Fabrigar et al., 1999), c) the items loaded on the same factor should be similar with regard to the theoretical concept. Due to the nature of multivariate analysis, after eliminating each item, the values of factor loadings changed. Therefore, we had to be careful about each item removed; subsequently EFA was conducted until the criteria of factor interpretation were satisfied. After the seventeenth elimination of poorly performing items, 23 items were retained.

We conducted further EFA on the final 23-items of the Workplace Forgiveness Scale. The correlation matrix of 23-items on Workplace Forgiveness Scale had good factorability, Bartlett's test of sphericity showed the chi-square was significant at the .0001 level (Chi-square $=3987.88, d f=253$ ) and the index of Kaiser-Mayer-Olkin measure of sampling adequacy was equal to .89 . Results from a parallel analysis indicated a dominant four-factor solution of the retained 23-items of the Workplace Forgiveness Scale, with factor I, II, III, and IV explaining 32.04, 12.79, 8.19 , and 6.94 percent of the variance, respectively. The four factors accounted for 59.95 percent of the variance in the Workplace Forgiveness Scale.

Finding from Varimax rotation showed that the factor loadings belonging to each factor were satisfied, see Table 1. The labelled numbers of items were re-arranged ( $f 1$ to $f 23$ ). The variables linked to factor I were named Overcoming Negative Thought and Feeling towards the Offender (ON; 6 variables labelled as $f 1$ to $f 6$ ), variables linked to factor II - Seeking to Understanding the Offender's Reasons (SR; 4 variables labelled as $f 7$ to $f 10$ ), variables linked to factor III - Fostering Positive Approaches towards the Offender (FP; 8 variables labelled as f11 to f18), and variables linked to factor IV - Belief in the Benefits of Forgiveness (BB; 5 variables labelled as f19 to f23).

As Shown in Table 2, the total Workplace Forgiveness Scale correlated significantly at .01 level with its four subscales, these correlation coefficients were $.75, .45, .87$, and .66 respectively. The mean scores of the total Forgiveness Scale and its four subscales were high. The highest subscale's mean was Belief in the Benefits of Forgiveness and the lowest among the subscales was Seeking to Understanding the Offender's Reasons. The intercorrelations between the Workplace Forgiveness Scale, its subscales, and other variables in this study are presented in Table 2. 


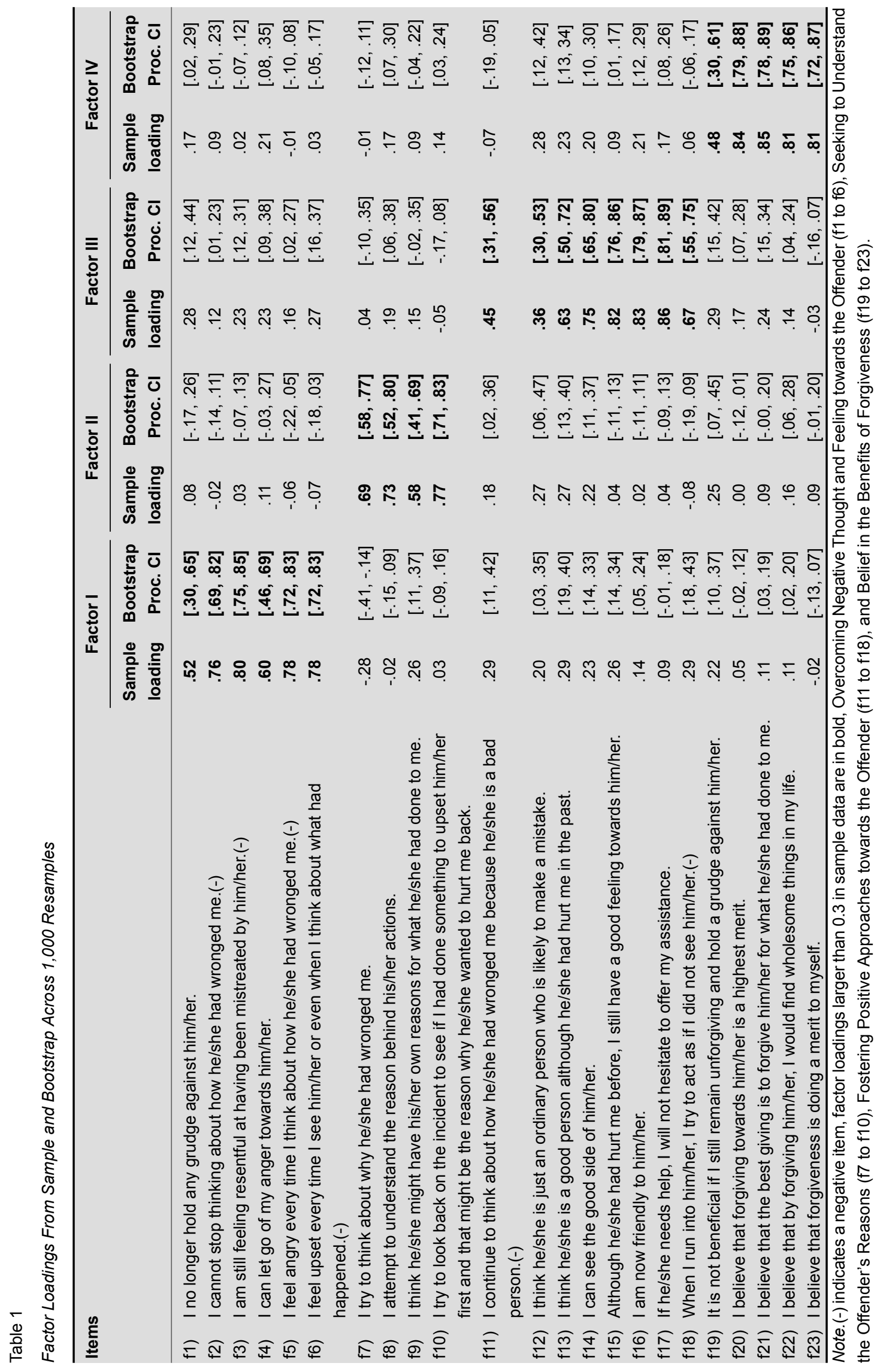


Table 2

Correlation Coefficients, Reliability, Mean, and Standard Deviations of Measures

\begin{tabular}{|c|c|c|c|c|c|c|c|c|c|c|c|c|c|c|}
\hline & Variables & 1 & 2 & 3 & 4 & 5 & 6 & 7 & 8 & 9 & 10 & 11 & 12 & 13 \\
\hline 1. & Overall WFS & $(.89)$ & & & & & & & & & & & & \\
\hline 2. & ON & $.75^{\star *}$ & $(.85)$ & & & & & & & & & & & \\
\hline 3. & SR & $.45^{\star *}$ & .04 & $(.68)$ & & & & & & & & & & \\
\hline 4. & $\mathrm{FP}$ & $.87^{\star *}$ & $.55^{\star *}$ & $.24^{\star *}$ & $(.87)$ & & & & & & & & & \\
\hline 5. & BB & $.66^{* *}$ & $.27^{* *}$ & $.29^{* *}$ & $.42^{\star *}$ & $(.85)$ & & & & & & & & \\
\hline 6. & Specific-Offense Forgiveness & $.64^{* *}$ & $.63^{* *}$ & $.12^{*}$ & $.53^{* *}$ & $.40^{* *}$ & $(.83)$ & & & & & & & \\
\hline 7. & Dispositional Forgiveness & $.63^{* *}$ & $.49^{* *}$ & $.25^{\star *}$ & $.52^{* *}$ & $.46^{* *}$ & $.64^{* *}$ & $(.67)$ & & & & & & \\
\hline & State Forgiveness & $.56^{* *}$ & $.50^{* *}$ & .08 & $.53^{* *}$ & $.33^{\star *}$ & $.56^{\star *}$ & $.49^{\star *}$ & $(-)$ & & & & & \\
\hline & Positive Affect & $.18^{* *}$ & $.12^{*}$ & $.14^{* *}$ & .06 & $.25^{\star *}$ & $.25^{\star *}$ & $.22^{\star *}$ & $.21^{\star *}$ & $(.91)$ & & & & \\
\hline & Negative Affect & $-.17^{* *}$ & $-.25^{* *}$ & .01 & -.09 & -.08 & $-.32^{* *}$ & $-.21^{\star *}$ & -.10 & $-.12^{*}$ & $(.70)$ & & & \\
\hline & Willingness to Reconcile & $.54^{\star *}$ & $.37^{* *}$ & $.16^{* *}$ & $.62^{* *}$ & $.22^{* *}$ & $.43^{* *}$ & $.38^{* *}$ & $.41^{\star *}$ & $.11^{*}$ & -.05 & $(.86)$ & & \\
\hline 12 & Rumination & $-.37^{* *}$ & $-.50^{* *}$ & .07 & $-.26^{\star *}$ & $-.22^{* *}$ & $-.64^{* *}$ & $-.40^{* *}$ & $-.40^{* *}$ & $-.17^{* *}$ & $.35^{* *}$ & $-.14^{* *}$ & $(.88)$ & \\
\hline & Seeking Revenge & $-.53^{* *}$ & $-.43^{* *}$ & $-.13^{*}$ & $-.50^{* *}$ & $-.32^{* *}$ & $-.63^{* *}$ & $-.62^{* *}$ & $-.47^{\star *}$ & $-.16^{* *}$ & $.21^{\star *}$ & $-.41^{* *}$ & $.59^{* *}$ & $(.95)$ \\
\hline$M \epsilon$ & & 4.30 & 3.92 & 4.32 & 4.15 & 5.01 & 3.40 & 5.07 & 3.90 & 3.90 & 2.30 & 3.04 & 2.48 & 2.13 \\
\hline$S D$ & & 0.60 & 0.92 & 0.80 & 0.82 & 0.79 & 0.50 & 0.88 & 0.93 & 0.72 & 0.63 & 0.83 & 0.79 & 0.89 \\
\hline
\end{tabular}

Note: Cronbach's Alphas were shown in parentheses, State Forgiveness has one item. WFS = Workplace Forgiveness Scale. ${ }^{*} p<.05 .{ }^{* *} p<.01$.

\section{Bootstrapping the Results From Exploratory Factor Analysis}

Bootstrapping is an effective statistical method for examining the stability and replicability of results (Guthrie, 2001). Several researchers proposed bootstrap methods to confirm the results from factor analysis (Raykov \& Little, 1999; Timmerman et al., 2007; Zientek, 2006; Zientek \&Thompson, 2007).We first aimed to investigate the internal replicability of the number of factors and whether the bootstrapped result yielded four factors. Hence, the bootstrapped eigenvalue employed by Zientek and Thompson (2007) was conducted to assure the correct number of factors to retain. Using a thousand resamples of bootstrapping, we generated the mean eigenvalues for each factor and created a distribution resulting in the standard deviations being the estimated standard error of the eigenvalues (Thompson, 1996). Consequently, the estimates can determine whether the mean of bootstrapped eigenvalues is greater than 1 and whether the SEs of mean bootstrapped eigenvalues are large or small (Zientek \& Thompson, 2007). Bootstrapped SEs provide the researchers with the concept of stability of the eigenvalues over a thousand times of resampling (Guthrie, 2001), representing the internal replicability of the number of factors to retain. Figure 1 showed the plots of empirically estimated sampling distributions for the eigenvalues. The first eigenvalue was 7.43 , ranging from 6.27 to 9.03 (95\% $\mathrm{Cl}[6.61,8.32])$. The second eigenvalue was 2.99 , ranging from 2.38 to $3.64(95 \% \mathrm{Cl}[2.60,3.39])$. The third eigenvalue was 1.98 , ranging from 1.50 to 2.67 (95\% $\mathrm{Cl}[1.66$, 2.38]). The fourth eigenvalue was 1.59 , ranging from 1.25 to $1.99(95 \% \mathrm{Cl}[1.37,1.83])$.

Of particular notice was the ambiguous result from the fifth eigenvalue. The sample estimate showed the fifth eigenvalue was lower than 1 (0.97) but the mean bootstrapped estimate was 1.05 , raising uncertainty about this factor. Zientek (2006) suggests using the range of estimates to decide on factor extraction. The fifth eigenvalue ranged from .86 to 1.40 . Of the 1000 resampling results, 23.80 percent of the fifth eigenvalue were smaller than one. Moreover, $95 \%$ percentile confidence interval was .92 to 1.96 indicating the lower level of confidence interval was less than 1 . Therefore, we were not confident with the fifth factor and concluded that a four factor model of forgiveness was more stable. Consequently, the bootstrap findings confirmed the results from EFA, representing good replicability of the four factors model of the 23-items of the Workplace Forgiveness Scale.

Europe's Journal of Psychology

2013, Vol. 9(2), 319-338

doi:10.5964/ejop.v9i2.551 


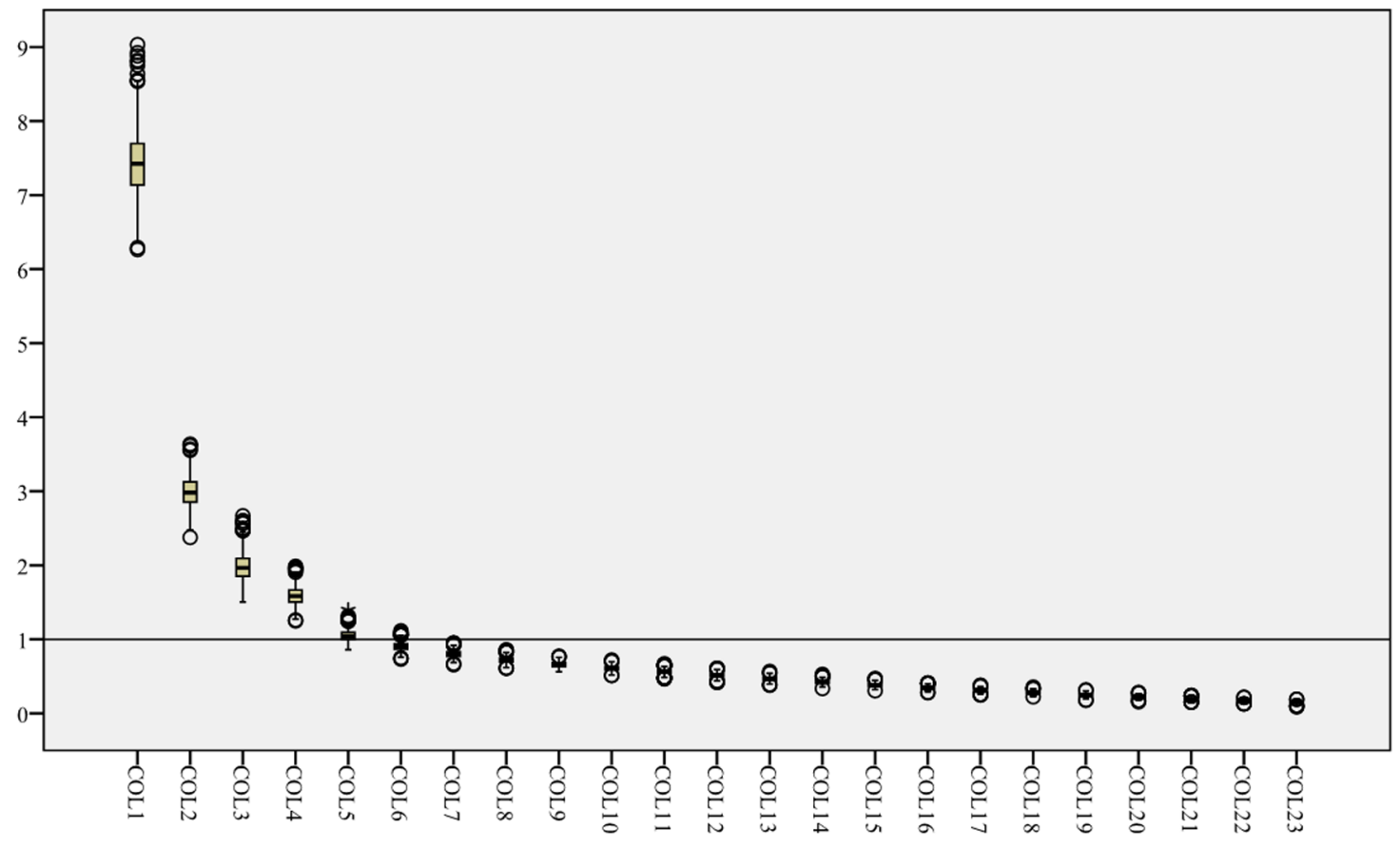

Figure 1. Empirically estimated sampling distribution of the 23 eigenvalues.

Procrustes rotation of factor loading matrix was then implemented to obtain Cls for loadings in EFA, generalizing the sample results across samples as Zientek and Thompson (2007) suggest that any other rotation solutions may show incorrect results. The optimal interpretability of the Procrustes approach using a target matrix in order to conduct the bootstrapping on the component loading, resulted in better Cls performance (Timmerman et al., 2007). For the present study, the target matrix was created, as a prior given loading matrix, from the sample factor loading matrix of 23-items. We followed the approach obtaining bootstrap procrustes confidence interval from Timmerman et al. (2007). Each bootstrap loading matrix was rotated using orthogonal Procrustes rotation (Cliff, 1966) with fixed four components. Results from 1000 bootstrappings achieved an empirically estimated distribution, where Cls were estimated. The coverage of bootstrap Cls on sample factor loading determined the stability of the sample estimates across the samples. Results revealed all of the sample's factor loadings were covered by the corresponding lower and upper ends of 95 percent BCa Procrustes confidence intervals, and the lower Cls of bootstrapped factor loadings are all over .30, indicating replicability and stability of the results of interpreted items from the sample EFA (see Table 1). Moreover, with the four factors structure, bootstrapped $95 \%$ confidence interval for the proportion of explained variance ranged from .56 to .62 , covering the explained variance from the sample (.5995). As a result, we concluded that the 23 items of the forgiveness scale would be stable if a new sample was obtained, yielding good internal replicability of the scale.

\section{Reliability Analyses}

Two types of reliability analyses were implemented as recommended by Gu, Little, and Kingston (2009). First is the internal consistency of Alpha reliability coefficient ( $\alpha$; Cronbach, 1951) and the second is Raykov's composite reliability ( $\rho$; Raykov, 1997), which structural equation modelling was used to provide more precise reliability estimate (Fan, 2003). The reliability coefficients for the overall Workplace Forgiveness Scale ( $\alpha=.89 ; \rho=.88)$, factor I $(\alpha=.85 ; \rho=.86)$, factor III $(\alpha=.87 ; \rho=.88)$, and factor IV $(\alpha=.85 ; \rho=.86)$ yielded good reliability. Reliability 
coefficient for Factor II was lower than .70, ( $\alpha=.68 ; \rho=.69)$, nevertheless, it was deemed satisfactory ( $\alpha$ between $.60-.70)$ as mentioned by Aiken (2000).

\section{Evidence of Construct Validity}

Construct Validity of a scale refers to the extent to which the scale measures a particular construct or psychological concept (Aiken, 2000). To examining construct validity requires a complex process, determining a variety of evidence to assess the extent to which scores on quantitative scales reveal the respondent's standing on the construct of interest (Hoyt, Warbasse, \& Chu, 2006).

Convergent Validity. Campbell and Fiske (1959) suggested that evidence of convergent validation should be employed to reveal construct validity. When the score on the given scale correlates moderately or highly with score on the theoretically concerned construct, this identifies as convergent validity. In the scale development process, it is important to demonstrate convergent validity of a measure with other instruments that have known properties (Anastasi, 1988; Cronbach \& Meehl, 1955). We would like to answer thus the question of whether the 23-items of the forgiveness scale correlate or converge with other standard measures of the forgiveness construct. To assess this validity, we created the mean of the composite score of 23-items representing the Workplace Forgiveness Scale. This score is then expected to be positively correlated with three other forgiveness measures, specific-offensive forgiveness (Rye et al., 2001), dispositional forgiveness (Yamhure-Thompson \& Snyder, 2003), and the single item of State forgiveness. Results revealed satisfactory evidence of convergent validity for the Workplace Forgiveness Scale. Scores from the Workplace Forgiveness Scale were positively associated with specific-offensive forgiveness $(r=.64, p<.01)$, indicating that participants rating themselves highly on the Workplace Forgiveness Scale tend to forgive on the specific-offensive scale as well. The Workplace Forgiveness Scale was positively associated with dispositional forgiveness $(r=.63, p<.01)$, indicating that the person who has a high score on this scale is likely to forgive others in general. The Workplace Forgiveness Scale was positively correlated with state forgiveness $(r=.56, p<.01)$, indicating that individuals who have a high score on the forgiveness scale tend to rate higher on their decision to forgive their offender within their specific work relationship conflict. Moreover, the bootstrapped correlation coefficients of 2,000 resamples revealed that all the empirical estimates of standard error were small (ranged from .03 to .04 ) and the $\mathrm{BC}_{\mathrm{a}} 95 \%$ confidence interval analysis yielded the moderate positive correlations between the Workplace Forgiveness Scale and specific-offensive forgiveness $(.57, .71)$ for the Workplace Forgiveness Scale and dispositional forgiveness $(.55, .70)$, and for the Workplace Forgiveness Scale and state forgiveness $(.47, .63)$. These bootstrap results revealed the stability of convergent validity of the Workplace Forgiveness Scale across the samples. The findings provide initial evidence of the convergent property of the Workplace Forgiveness Scale with two psychometrically sound instruments measuring forgiveness and a single item of state forgiveness representing the consistency between the score on multi-items measure of forgiveness and specific decision on forgiveness towards the offender, yielding similar results to those found by McCullough et al. (1998).

Discriminant Validity. As mentioned by Churchill and lacobucci (2002), we predicted that the score on the Workplace Forgiveness Scale would not be highly correlated with the PANAS measure, based on previous research. Small correlations were found with the measure of positive affect $(r=.18, p<.01)$ and the measure of negative affect $(r=-.17, p<.01)$. The bootstrapped correlation coefficients 2,000 resamples revealed that all the empirically estimates of standard error were small (.05 and .06, respectively) and the $\mathrm{BC}_{\mathrm{a}} 95 \%$ confidence interval analysis yielded the small correlations between the Workplace Forgiveness Scale and positive affect $(.05, .31)$, the Workplace Forgiveness Scale and negative affect (-.27, -.07). The finding of discriminant evidence is consistent with McCullough 
et al. (1998), who examined the discriminant validity of the offense-specific forgiveness scale (TRIM) with positive and negative affect.

Nomological Validity. Researchers must achieve a nomological network linkage between the construct intended to be validated and other variables, which have been proven theoretically related (Cronbach \& Meehl, 1955). "Nomological validity", as presented in Viswanathan (2005) and Hair et al. (2006), refers to the structural relationship model of the specified construct and related variables. We would like thus to answer the question of whether the forgiveness construct behaves in a theoretically expected way. Two mediation models were proposed to examine the theoretical network of the forgiveness construct and other psychological constructs.

The first mediation model hypothesised that dispositional forgiveness would be positively associated with forgiveness for the specific-offensive event (Koutsos, Wertheim, \& Kornblum, 2008; Wade \& Worthington, 2003) and the increase of forgiveness would then contribute to a willingness to reconcile with the offender (Aquino et al., 2003). We conducted mediation analysis following the steps suggested by Frazier, Tix, and Barron (2004), conducting three regression analyses. In the first analysis, willingness to reconcile was regressed on dispositional forgiveness achieving the coefficient corresponding to Path $c$ in Table 3. The unstandardized regression coefficients of path $c$ was $.36(p<.01)$. The second step was that forgiveness, as the mediator variable, was regressed on dispositional forgiveness to obtain the regression coefficient for Path $a$. The unstandardized regression coefficients for Path a was $.43(p<.01)$. In the third step, willingness to reconcile was regressed simultaneously on both forgiveness and dispositional forgiveness. This analysis provided the unstandardized regression coefficients for Path $b$ and Path $c^{\prime}$. These were $.69(p<.01)$ and .06 , respectively. The unstandardized regression coefficients were divided by the standard error yielding a $Z$ statistic that could be used for statistical significance in the Normal Theory approach (Frazier et al., 2004). Results shown Path $a, b$, and $c$ were satisfied, critical ratio values were 15.32, 8.66 and 7.10 , respectively. However, the regression coefficient of $c^{\prime}$ was relatively small and did not reach significance due to the critical ratio being 1.05 .

Table 3

Evidence of Nomological Validity by Testing Two Mediation Models With Forgiveness as a Mediator

\begin{tabular}{|c|c|c|c|c|c|c|}
\hline \multirow[b]{2}{*}{ Path/effect } & \multicolumn{2}{|c|}{ Sample Regression result } & \multicolumn{2}{|c|}{ Bootstrap estimate } & \multicolumn{2}{|c|}{$\begin{array}{c}\text { Bootstrap BCa } 95 \% \text { Confidence } \\
\text { interval }\end{array}$} \\
\hline & B & $S E$ & $\bar{B}$ & $\overline{S E}$ & Lower & Upper \\
\hline \multicolumn{7}{|c|}{ First Mediation Model (Dfg $\rightarrow$ Fg $\rightarrow$ Reconcile) } \\
\hline$c($ Dfg $\rightarrow$ Reconcile $)$ & $.36^{* *}(.38)$ & .05 & $.35(.38)$ & .05 & .26 & .45 \\
\hline$a(\mathrm{Dfg} \rightarrow \mathrm{Fg})$ & $.43^{* *}(.63)$ & .03 & $.43(.63)$ & .03 & .36 & .49 \\
\hline$b(\mathrm{Fg} \rightarrow$ Reconcile $)$ & $.69^{* *}(.50)$ & .08 & $.69(.50)$ & .10 & .49 & .88 \\
\hline$c^{\prime}$ & $.06(.06)$ & .05 & $.06(.06)$ & .07 & -.07 & .20 \\
\hline$a \times b$ & $.30^{* *}(.32)$ & .04 & $.30(.32)$ & .05 & .20 & .40 \\
\hline \multicolumn{7}{|c|}{ Second Mediation Model (Rumi $\rightarrow$ Fg $\rightarrow$ Revenge) } \\
\hline$c$ (Rumi $\rightarrow$ Revenge) & $.67^{\star *}(.59)$ & .05 & $.67(.59)$ & .06 & .54 & .78 \\
\hline a (Rumi $\rightarrow \mathrm{Fg})$ & $-.28^{\star *}(-.37)$ & .04 & $-.28(-.37)$ & .05 & -.37 & -.19 \\
\hline$b(\mathrm{Fg} \rightarrow$ Revenge $)$ & $-.54^{\star *}(-.36)$ & .06 & $-.53(-.36)$ & .09 & -.72 & -.36 \\
\hline$c^{\prime}$ & $.52^{* *}(.46)$ & .04 & $.52(.46)$ & .07 & .39 & .65 \\
\hline$a \times b$ & $.15^{\star *}(.13)$ & .03 & $.15(.13)$ & .04 & .10 & .22 \\
\hline
\end{tabular}

Note. Standardised estimates are in the round brackets, Dfg = Dispositional forgiveness, Reconcile = Willingness to reconcile, Rumi = Rumination, Revenge = Seeking revenge, $\mathrm{Fg}=$ Forgiveness (as measured by the Workplace Forgiveness Scale in this study).

${ }^{* *} p<.01$. 
To examine the indirect effect of dispositional forgiveness on willingness to reconcile, the unstandardized regression coefficient of a was multiplied by $b$ resulting $a \times b$ was .30 and the standard error of $a \times b$ was calculated followed by Baron and Kenny (1986) using

$$
\sqrt{a^{2} s b^{2}+b^{2} s a^{2}+s a^{2} s b^{2}}
$$

where sa was the standard error of $a$ and $s b$ was the standard error of $b$. Using this formula, standard error of $a$ $x b$ was .04 . The critical ratio of $a x b$ was 7.62 indicating statistical significance and that mediation had occurred. On the basis of this method, we concluded that the indirect effect of dispositional forgiveness on willingness to reconcile was mediated by forgiveness.

Finally, to investigate the mediator role of forgiveness, the regression coefficient of $c$ and $c^{\prime}$ were compared (Frazier et al., 2004). The relation between dispositional forgiveness and willingness to reconcile ( $c^{\prime}$ ) did not differ from zero, or was not significant, after forgiveness was included in the model. This result yielded the fully mediating role of forgiveness on the relationship between dispositional forgiveness and willingness to reconcile.

In order to examine the internal replicability of the sample results, we conducted the bootstrap method of mediation analysis described by Mallinckrodt, Abraham, Wei, and Russell (2006), using the AMOS program. The bootstrap option was selected requesting 2,000 resamples with $\mathrm{BC}_{\mathrm{a}} 95 \%$ percentile confidence interval. The result showed that the means of bootstrapped estimates $(\bar{B})$ slightly differed from the sample estimates (see Table 3 ). The means standard error of estimates based on 2,000 empirical samples were relatively small. The means of estimate (and means of standard error) of the bootstrapping were $\hat{a}=.43(.03), \hat{b}=.69(.10), \hat{c}=.35(.05)$, and $\hat{c}^{\prime}=.06$ (.07). The bootstrap 95\% confidence interval excluded zero for $a, b$, and $c$, achieving statistically significance by conventional standards (Shrout \& Bolger, 2002). The 95\% confidence interval for $a \times b$ ranged from .20 and .40 , indicating that the indirect effect was occurring. The $95 \%$ confidence interval of $c^{\prime}$ was around -.07 to .20 with the range of $\mathrm{Cl}$ including zero, showing forgiveness played a fully mediating role in the relationship between dispositional forgiveness and willingness to reconcile. Results from the bootstrapping supported the stability of the mediation analysis results across the samples.

Investigating the second nomological network of the forgiveness construct, we hypothesised that rumination would be negatively related to forgiveness for the specific-offensive event (Burnette, Davis, Green, Worthington, \& Bradfield, 2009; Greenberg, 1995; McCullough, Bono, \& Root, 2007) and that it is this lack of forgiveness that contributes to the intention to seek to take revenge on the offender (McCullough, Bellah, Kilpatrick, \& Johnson, 2001). A mediation model with forgiveness as a mediator between rumination and seeking revenge was tested (see Table 3). In the first analysis, seeking revenge was regressed on rumination achieving the coefficient corresponding to Path $c$. The unstandardized regression coefficient of path $c$ was $.67(p<.01)$. The second step was that forgiveness, as the mediator variable, was regressed on rumination to obtain the unstandardized regression coefficient for Path $a$, which was $-.28(p<.01)$. In the third step, seeking revenge was regressed simultaneously on both forgiveness and rumination. This analysis provided the unstandardized regression coefficients for Path $b$ and $c^{\prime}$. These were $-.54(p<.01)$ and $.52(p<.01)$, respectively. Results shown Path $a, b, c, c^{\prime}$ were statistical significance, critical ratio values were $-7.36,-8.52,13.36$, and 10.79 , respectively.

Regarding the indirect effect of rumination on seeking revenge, the unstandardised regression coefficient of $a x$ $b$ was .15 and the standard error of $a x b$ was .03 . The critical ratio of $a \times b$ was 5.56 indicating statistical significance and indicating that mediation had occurred. On the basis of this method, we concluded that the indirect effect of 
rumination on seeking revenge was mediated by forgiveness. To investigate the mediator role of forgiveness, the relation between rumination and seeking revenge $\left(c^{\prime}\right)$ was smaller with statistical significance at .01 level, after forgiveness was included in the model. This result yielded the partial mediator role of forgiveness on the relationship between rumination and seeking revenge.

Bootstrapping the second mediation model was undertaken and the results show that the means of the bootstrapped estimates $(\bar{B})$ slightly differed from the sample estimates. The means of the estimate (and means of standard error) of the bootstrapping were $\hat{a}=-.28(.05), \hat{b}=-.53(.09), \hat{c}=.67(.06)$, and $\hat{c}^{\prime}=.52$ (.07). The bootstrap 95\% confidence interval excluded zero for $a, b, c$, and $c$ 'achieving the statistical significance by conventional standards. The $95 \%$ confidence interval for $a \times b$ ranged from .10 and .22 , indicating that the indirect effect was occurring. The $95 \%$ confidence interval of $c^{\prime}$ was around .39 to .65 which excluded zero, showing forgiveness played a partial mediating role in the relationship between rumination and seeking revenge. Results from the bootstrapping yielded the stability of mediation analysis results across the samples.

In conclusion, results from two mediation analyses yielded empirical evidence representing the good nomological validity of the scale. Using both the convergent validity evidence and nomological validity evidence, we could be confident that the forgiveness construct measured by the 23-items of the forgiveness scale developed in this study is psychometrically sound, achieving construct validity. Moreover, results from the bootstrapping also showed internal replicability thus assuring the stability of the results across samples.

\section{Discussion}

Achieving a psychometrically sound scale designed to measure forgiveness in workplace relationships within a Buddhist culture will provide the means for further research in this context. A four-factor underlying structure of forgiveness emerged from an exploratory factor analysis as representing the forgiveness construct empirically identified by Thai professional nurses within their work situation. These are overcoming negative thought and feeling towards the offender, seeking to understand the offender's reasons, fostering positive approaches towards the offender, and belief in the benefits of forgiveness. This finding confirms the definition of forgiveness that emerged from Boonyarit et al. (2012) where forgiveness was seen as an individuals' readiness to overcome their negative thoughts and emotions, attempting to relinquish their negative judgment, and instead offering more positive views, feelings, and acts towards the offender.

The first factor, overcoming negative thought and feeling towards the offender is consistent with the concept as described in the existing literature (Aquino et al., 2003; Enright \& Coyle, 1998; McCullough et al., 2000; Worthington, 1998) in that, when individuals forgive people who hurt them, they try to overcome their destructive thoughts and let go of their negative feeling such as resentment, anger, hostility towards the offenders. Fostering positive approaches towards the offender is also consistent with ideas from previous literature (Enright \& Coyle, 1998; Hargrave \& Sell, 1997; McCullough et al., 2000; Worthington, 1998) which infer that forgiveness is a prosocial change in one's view, feeling, and action towards an offending relationship partner. The individual offers more positive thoughts and feelings, empathy, and continues to act in a friendly manner with their offender. Furthermore, the factor identified as seeking to understand the offender's reasons is consistent with a step within the work phase of forgiveness mentioned by Enright, Freedman, and Rique (1998). After being hurt, individuals attempt to understand the offender's reasons such as their personal issues and present pressures. This factor is an instrumental dimension of forgiveness, involving relinquishing blame towards the offender. The final factor, belief in the benefits 
of forgiveness, is a combination of awareness of the benefits of forgiveness and forgiveness as Buddhist beliefs, as found in Boonyarit et al. (2012). This factor is seen to be salient representing both the prosocial motivation of forgiveness as suggested by McCullough, Worthington, and Rachal (1997) and the influence of Buddhist culture on the forgiveness concept among the participants (Rye et al., 2000). It demonstrates that individuals foresee the positive consequences of forgiveness as being a good choice in dealing with their relationships, and it is consistent with Buddhist beliefs about individuals forgiving others as doing a good merit or positive Karma (Phra Brahmagunabhorn, 2007).

Evidence of nomological validity from the two mediation analyses revealed the theoretical network of forgiveness. Specific offensive forgiveness, as measured by the 23-items scale, was positively related to dispositional forgiveness, consistent with findings from Wade and Worthington (2003), and Koutsos et al. (2008). Moreover, it was positively correlated with willingness to reconcile. This result confirms the concept of forgiveness and its consequences in the workplace suggested by Aquino et al. (2003): increasing forgiveness would contribute to maintain work relationships. The fully mediating role of specific offense forgiveness on the relationship between dispositional forgiveness and willingness to reconcile reveals the systematic interplay between dispositional forgiveness, specific offensive forgiveness, and reconciliation as theorised by McCullough et al. (1998). According to their proposition, dispositional or trait forgiveness is the distal determinant facilitating forgiving towards the offender in casual chains, then forgiving in relational offenses might contribute to restore the relationship between conflicting partners, being as identified as a willingness to reconcile in this study. The distal role of dispositional variables in the forgiveness mediation process (McCullough et al., 1998; Shrout \& Bolger, 2002) yields answers as to why the relationship between dispositional forgiveness and willing to reconcile becomes smaller and not significant after including specific offensive forgiveness in the model.

The second nomological network evidence shows the negative relationship between rumination and forgiveness in the specific offensive event, being consistent with previous findings from Burnette et al. (2009), Greenberg (1995), and McCullough et al. (2007) that rumination is negatively related to an individual's decision to forgive the offender. Forgiveness was negatively related to the intention to seek revenge against the offender. This is consistent with the correlation results found from McCullough et al. (2001). The partial mediating role of specific offense forgiveness on the relationship between rumination and seeking revenge reveals the linkage between rumination, specific offensive forgiveness, and seeking revenge. In the causal chain described by McCullough et al. (1998), rumination is the social-cognitive determinant of forgiving specific relationship partners. Repetitive thought about the offensive event is the most proximal predictor of forgiveness, more rumination results and less forgiveness towards the offender. Moreover, individuals who cognitively ruminate about the event also maintain their motivation to seek revenge towards his or her wrongdoer. The proximal relationship between rumination and forgiveness, and rumination and seeking revenge might yield the partial mediating role of forgiveness in this model.

\section{Implications}

The findings from this research provide a deeper understanding of workplace related forgiveness. The EFA revealed that four factors related to workplace forgiveness can be established. Factor I, II, and III seem to be consistent with the forgiveness' subscales in the western measures. However, the last factor, Belief in the Benefits of Forgiveness, demonstrated Buddhist Thai views of forgiveness as having benefits and positive gains for the individual granting forgiveness. The motivational concept of workplace forgiveness thus revealed the role of culture in the 
forgiveness process within a work context. Further research should clarify how culture and Buddhist belief shape people's cognition and behaviour regarding forgiveness and coping with conflict at work.

The practical implication is that the Workplace Forgiveness Scale can be used by counselors or human resource coaches as a tool whenever they consider forgiveness a constructive way of coping with an interpersonal conflict. This measure illustrates clients' cognition, affects, and behavior towards the offenders and gives them a measure of the current level of forgiveness. By using this measure coupled with forgiveness counseling, the clients' progress towards forgiveness can be assessed.

Moreover, the results of the present study have implications for future research. Confirmatory factor analysis should be implemented in order to validate the underlying structure resulting from the exploratory factor analysis, confirming the construct validity of the four factors model of forgiveness derived from the scale. Given the limitation of a single sample analysis of the results, research in additional healthcare or other work contexts should be conducted. Further studies with different work populations are required to assess the generalizability of the measure. Moreover, in the present study, we conducted an internal replicability using the bootstrap method. This provides evidence of the stable psychometric properties of the forgiveness scale; however, it is not a true replication analysis. Further external replicability (Thompson, 1994) should be implemented by collecting data from a new sample. This notwithstanding, to extend the nomological network of the forgiveness construct within the work context, future research should include more work-related variables. For instance, further research should examine the role of work related socio-cultural factors such as seniority and the status of the offender in the decision to forgive, the constructive or non-constructive behaviours related to forgiveness or un-forgiveness, the work outcomes resulting from forgiveness such as performance, cohesiveness, and team climate. A final limitation of this research is that participants were mostly female nurses; future research should compare the differences in granting forgiveness between female and male employees.

\section{Funding}

The authors would like to thank the Office of the Higher Education Commission, Thailand, for supporting by grant fund under the program Strategic Scholarships for Frontier Research Network for the Joint Ph.D. Program, Thai Doctoral Degree, for this research.

\section{References}

Aiken, L. R. (2000). Psychological testing and assessment (10th ed.). Needham Heights: Allyn and Bacon, Inc.

Anastasi, A. (1988). Psychological testing (6th ed.). New York: MacMillan Publishing Company.

Aquino, K., Grover, S. L., Goldman, B., \& Folger, R. (2003). When push doesn't come to shove: Interpersonal forgiveness in workplace relationships. Journal of Management Inquiry, 12(3), 209-216. doi:10.1177/1056492603256337

Baron, R. M., \& Kenny, D. A. (1986). The moderator-mediator variable distinction in social psychological research: Conceptual, strategic, and statistical considerations. Journal of Personality and Social Psychology, 51, 1173-1182.

doi:10.1037/0022-3514.51.6.1173

Bartlett, M. S. (1954). A note on the multiplying factors for various $x^{2}$ approximation. Journal of the Royal Statistical Society, Series B: Methodological, 16, 296-298. 
Boonyarit, I., Chuawanlee, W., Macaskill, A., \& Supparerkchaisakul, N. (2012). Thai conceptualizations of forgiveness within a work context: Comparison with Western models. International Journal of Behavioral Science, 7(1), 1-30.

Burnette, J. L., Davis, D. E., Green, J. D., Worthington, E. L., Jr, \& Bradfield, E. (2009). Insecure attachment and depressive symptoms: The mediating role of rumination, empathy, and forgiveness. Personality and Individual Differences, 46, $276-280$. doi:10.1016/j.paid.2008.10.016

Butler, D. S., \& Mullis, F. (2001). Forgiveness: A conflict resolution strategy in the workplace. Journal of Individual Psychology, 57(3), 259-272.

Cameron, K., \& Caza, A. (2002). Organizational and leadership virtues and the role of forgiveness. Journal of Leadership \& Organizational Studies, 9(1), 33-48. doi:10.1177/107179190200900103

Campbell, D. T., \& Fiske, D. W. (1959). Convergent and discriminant validity by the multitrait-multimethod matrix. Psychological Bulletin, 56(2), 81-105. doi:10.1037/h0046016

Churchill, G. A., \& lacobucci, D. (2002). Marketing research methodological foundations (8th ed.). Fort Worth: Harcourt College Publishers.

Cliff, N. (1966). Orthogonal rotation to congruence. Psychometrika, 31, 33-42. doi:10.1007/BF02289455

Costello, A. B., \& Osborne, J. W. (2005). Best practices in exploratory factor analysis: Four recommendations for getting the most from your analysis. Practical Assessment, Research \& Evaluation, 10(7). Retrieved from http://pareonline.net/getvn.asp?v=10\&n=7.

Cronbach, L. J. (1951). Coefficient alpha and the internal structure of tests. Psychometrika, 16, $297-334$. doi:10.1007/BF02310555

Cronbach, L. J., \& Meehl, P. E. (1955). Construct validity in psychological tests. Psychological Bulletin, 52, $281-302$. doi:10.1037/h0040957

Enright, R. D., \& Coyle, C. T. (1998).Researching the process model of forgiveness within psychological intervention. In E. L.Worthington (Ed.), Dimensions of forgiveness: Psychological research and theological perspectives (pp. 139-161). Philadelphia: Templeton Foundation Press.

Enright, R. D., Freedman, S., \& Rique, J. (1998). The psychology of interpersonal forgiveness. In R. D. Enright \& J. North (Eds.), Exploring forgiveness (pp. 46-63). Madison: University of Wisconsin Press.

Fabrigar, L. R., Wegener, D. T., MacCallum, R. C., \& Strahan, E. J. (1999). Evaluating the use of exploratory factor analysis in psychological research. Psychological Methods, 4(3), 272-299. doi:10.1037/1082-989X.4.3.272

Fan, X. (2003). Using commonly available software for bootstrapping in both substantive and measurement analyses. Educational and Psychological Measurement, 63(1), 24-50. doi:10.1177/0013164402239315

Frazier, P. A., Tix, A. P., \& Barron, K. E. (2004). Testing moderator and mediator effects in counseling psychology research. Journal of Counseling Psychology, 51(1), 115-134. doi:10.1037/0022-0167.51.1.115

Gorsuch, R. L. (1983). Factor analysis (2nd ed.). Hillsdale, NJ: Erlbaum. 
Greenberg, M. A. (1995). Cognitive processing of traumas: The role of intrusive thoughts and reappraisals. Journal of Applied Social Psychology, 25, 1262-1296. doi:10.1111/j.1559-1816.1995.tb02618.x

Gu, F., Little, T., \& Kingston, N. M. (2009, October). Using PROC CALIS and PROC CORR to compare structural equation modeling based reliability estimates and coefficient alpha when assumptions are violated. Paper presented at MWSUG 2009 Conference, Cleveland, Ohio. Retrieved from http://www.mwsug.org/proceedings/2009/stats/MWSUG-2009-D04.pdf

Guthrie, A. C. (2001, February). Using bootstrap methods with popular statistical programs. Paper presented at the annual meeting of the Southwest Educational Research Association, New Orleans.

Hair, J. F., Black, B.; Babin, B.; Anderson, R. E., \& Tatham, R. L. (2006). Multivariate data analysis (6th ed.). New Jersey: Prentice-Hall Inc.

Hargrave, T. D., \& Sell, J. N. (1997). The development of a forgiveness scale. Journal of Marital and Family Therapy, 23, 41-63. doi:10.1111/j.1752-0606.1997.tb00230.x

Horn, J. L. (1965). A rationale and technique for estimating the number of factors in factor analysis. Psychometrika, 30, 179-185. doi:10.1007/BF02289447

Hoyt, W. T., Warbasse, R. E., \& Chu, E. Y. (2006). Construct validation in counseling psychology research. The Counseling Psychologist, 34(6), 769-805. doi:10.1177/0011000006287389

Kaiser, H. F. (1974). An index of factorial simplicity. Psychometrika, 39, 31-36. doi:10.1007/BF02291575

Kline, P. (1986). A handbook of test construction: Introduction to psychometric design. New York, NY: Methuen.

Koutsos, P., Wertheim, E. H., \& Kornblum, J. (2008). Paths to interpersonal forgiveness: The roles of personality, disposition to forgive and contextual factors in predicting forgiveness following a specific offence. Personality and Individual Differences, 44, 337-348. doi:10.1016/j.paid.2007.08.011

Madsen, S. R., Gygi, J., Hammond, S. C., \& Plowman, S. (2008). Forgiveness as an HRD intervention: The literature and a proposed framework. Retrieved from http://works.bepress.com/susan_madsen/77/.

Mallinckrodt, B., Abraham, W. T., Wei, M., \& Russell, D. W. (2006). Advances in testing the statistical significance of mediation effects. Journal of Counseling Psychology, 53(3), 372-378. doi:10.1037/0022-0167.53.3.372

McCullough, M. E., Bellah, C. G., Kilpatrick, S. D., \& Johnson, J. L. (2001). Vengefulness: Relationship with forgiveness, rumination, well-being, and the big five. Personality and Social Psychology Bulletin, 27(5), 601-610.

doi:10.1177/0146167201275008

McCullough, M. E., Bono, G., \& Root, L. M. (2007). Rumination, emotion, and forgiveness: Three longitudinal studies. Journal of Personality and Social Psychology, 92(3), 490-505. doi:10.1037/0022-3514.92.3.490

McCullough, M. E., Pargament, K. I., \& Thoresen, C. E. (2000). The psychology of forgiveness: History, conceptual Issues, and overview. In M. E. McCullough, K. I. Pargament, \& C. E. Thoresen (Eds.), Forgiveness: Theory, research, and practice (pp. 1-14). New York: The Guilford Press.

McCullough, M. E., Rachel, K. C., Sandage, S. J., Worthington, E. L., Brown, S. W., \& Hight, T. L. (1998). Interpersonal forgiving in close relationships II: Theoretical elaboration and measurement. Journal of Personality and Social Psychology, 75, 1586-1603. doi:10.1037/0022-3514.75.6.1586

Europe's Journal of Psychology 
McCullough, M. E., \& Witvliet, C. V. O. (2002). The psychology of forgiveness. In C. R. Snyder \& S. J. Lopez (Eds.), Handbook of positive psychology (pp. 446-458). New York: Oxford University Press.

McCullough, M. E., \& Worthington, E. L., Jr. (1994). Models of interpersonal forgiveness and their applications to counseling: Review and critique. Counseling and Values, 39, 2-14. doi:10.1002/j.2161-007X.1994.tb01003.x

McCullough, M. E., Worthington, E. L., Jr., \& Rachal, K. C. (1997). Interpersonal forgiving in close relationships. Journal of Personality and Social Psychology, 73, 321-336. doi:10.1037/0022-3514.73.2.321

Phra Brahmagunabhorn. (2007). Tam yang rai ja hai grot [How to cure anger]. Bangkok: Sukapapjai.

Raykov, T. (1997). Scale reliability, Cronbach's coefficient alpha, and violations of essential tau-equivalence with fixed congeneric components. Multivariate Behavioral Research, 32(4), 329-353. doi:10.1207/s15327906mbr3204_2

Raykov, T., \& Little, T. D. (1999). A note on procrustean rotation in exploratory factor analysis: A computer intensive approach to goodness-of-fit evaluation. Educational and Psychological Measurement, 59(1), 47-57.

Rye, M. S., Loiacono, D. M., Folck, C. D., Olszewski, B. T., Heim, T. A., \& Madia, B. P. (2001). Evaluation of the psychometric properties of two forgiveness scales. Current Psychology, 20(3), 260-277. doi:10.1007/s12144-001-1011-6

Rye, M. S., Pargament, K. I., Ali, M. A., Beck, G. L., Dorff, E. N., \& Hallisey, C. ... Williams, J. G. (2000). Religious perspectives on forgiveness. In M. E. McCullough, K. I. Pargament, \& C. E. Thoresen (Eds.), Forgiveness: Theory, research, and practice (pp. 17-40). New York: The Guilford Press.

Shrout, P. E., \& Bolger, N. (2002). Mediation in experimental and nonexperimental studies: New procedures and recommendations. Psychological Methods, 7(4), 422-445. doi:10.1037/1082-989X.7.4.422

Stone, M. (2002). Forgiveness in the workplace. Industrial and Commercial Training, 34(7), 278-286. doi:10.1108/00197850210447282

Subkoviak, M. J., Enright, R. D., Wu, C.-R., Gassin, E. A., Freedman, S., Olson, L. M., \& Sarinopoulos, I. (1995). Measuring interpersonal forgiveness in late adolescence and middle adulthood. Journal of Adolescence, 18, 641-655. doi:10.1006/jado.1995.1045

Tabachnick, B. G., \& Fidell, L. S. (1996). Using multivariate statistics (3rd ed.). New York: HarperCollins College Publishers.

Thompson, B. (1994). The pivotal role of replication in psychological research: Empirically evaluating the replicability of sample results. Journal of Personality, 62, 157-176. doi:10.1111/j.1467-6494.1994.tb00289.x

Thompson, B. (1996, April). Problems with multivariate normality: Can the multivariate bootstrap help? Paper presented at the annual meeting of the Society for Applied Multivariate Research, Houston.

Thompson, E. R. (2007). Development and validation of an internationally reliable short-form of the Positive and Negative Affect Schedule (PANAS). Journal of Cross-Cultural Psychology, 38(2), 227-242. doi:10.1177/0022022106297301

Timmerman, M. E., Kiers, H. A., \& Smilde, A. K. (2007). Estimating confidence intervals for principal component loadings: A comparison between the bootstrap and asymptotic results. The British Journal of Mathematical and Statistical Psychology, 60, 295-314. doi:10.1348/000711006X109636

Europe's Journal of Psychology 
Tomlinson, E. C., Dineen, B. R., \& Lewicki, R. J. (2004). The road to reconciliation: Antecedents of victim willingness to reconcile following a broken promise. Journal of Management, 30(2), 165-187. doi:10.1016/j.jm.2003.01.003

Viswanathan, M. (2005). Measurement error and research design. Thousand Oaks: Sage.

Wade, N. G., Vogel, D. L., Liao, K. Y.-H., \& Goldman, D. B. (2008). Measuring state-specific rumination: Development of the rumination about an interpersonal offense scale. Journal of Counseling Psychology, 55(3), 419-426. doi:10.1037/0022-0167.55.3.419

Wade, N. G., \& Worthington, E. L., Jr. (2003). Overcoming interpersonal offense: Is forgiveness the only to deal with unforgiveness? Journal of Counseling and Development, 81, 343-353. doi:10.1002/j.1556-6678.2003.tb00261.x

Wade, S. H. (1987). A content analysis of forgiveness. (Unpublished master's thesis). Fuller Graduate School of Psychology, CA.

Watkins, M. W. (2006). Determining parallel analysis criteria. Journal of Modern Applied Statistical Methods, 5(2), 344-346.

Worthington, E. L., Jr. (1998). Empirical research on forgiveness: Looking backward, looking forward. In E. L. Worthington (Ed.), Dimensions of forgiveness: Psychological research and theological perspectives (pp. 321-339). Philadelphia: Templeton Foundation Press.

Yamhure-Thompson, L., \& Snyder, C. R. (2003). Measuring forgiveness. In S. J. Lopez \& C. R. Snyder (Eds.), Positive psychological assessment: A handbook of models and measures (pp. 301-312). Washington, DC: American Psychological Association.

Zientek, L. R. (2006). Do teachers differ by certification route? Novice teachers'sense of self-efficacy, commitment to teaching, and preparedness to teach (Unpublished doctoral dissertation). Texas A\&M University, Texas.

Zientek, L. R., \& Thompson, B. (2007). Applying bootstrap to the multivariate case: Bootstrap component/factor analysis. Behavior Research Methods, 39(2), 318-325. doi:10.3758/BF03193163

\section{About the Authors}

Dr. Itsara Boonyarit is a lecturer at Division of Psychology and postgraduate committee in Industrial and Organizational Psychology Program, Faculty of Humanities, Chiang Mai University. His research interests include applying positive psychology to work context, Buddhist psychology, psychology of risk, psychometrics, and organizational psychology.

Dr. Wiladlak Chuawanlee is an Assistant Professor (retired) and former Director, Behavioral Science Research Institute, Srinakharinwirot University, Thailand. Her primary interests focus on both psychology of work and educational psychology.

Dr. Ann Macaskill is a Professor of Health Psychology and Head of Research Ethics at Sheffield Hallam University. She is a Registered Practitioner Health Psychologist with the Health Professions Council, UK, a qualified psychotherapist, and a principal Fellow of the UK Higher Education Academy. Her research interests are in individual differences mainly in health and well-being, positive psychology and psychometrics. She has published over fifty peer reviewed journal articles, eight book chapters, a widely used textbook on individual differences, and a selfhelp book on forgiveness. 
Dr. Numchai Supparerkchaisakul is a Chair of Doctoral Program, Behavioral Science Research Institute, Srinakharinwirot University, Thailand, where he mainly teaches research methodology and statistics. His research interests include multilevel analysis of leadership and corporate image through societal marketing. 